\title{
TH-302 in Combination with Radiotherapy Enhances the Therapeutic Outcome and Is Associated with Pretreatment [F-18]HX4 Hypoxia PET Imaging
}

Citation for published version (APA):

Peeters, S. G. J. A., Zegers, C. M. L., Biemans, R., Lieuwes, N. G., van Stiphout, R. G. P. M., Yaromina, A., Sun, J. D., Hart, C. P., Windhorst, A. D., van Elmpt, W., Dubois, L. J., \& Lambin, P. (2015). TH-302 in Combination with Radiotherapy Enhances the Therapeutic Outcome and Is Associated with Pretreatment [F-18]HX4 Hypoxia PET Imaging. Clinical Cancer Research, 21(13), 2984-2992. https://doi.org/10.1158/1078-0432.CCR-15-0018

Document status and date:

Published: 01/07/2015

DOI:

10.1158/1078-0432.CCR-15-0018

Document Version:

Publisher's PDF, also known as Version of record

Document license:

Taverne

Please check the document version of this publication:

- A submitted manuscript is the version of the article upon submission and before peer-review. There can be important differences between the submitted version and the official published version of record.

People interested in the research are advised to contact the author for the final version of the publication, or visit the DOI to the publisher's website.

- The final author version and the galley proof are versions of the publication after peer review.

- The final published version features the final layout of the paper including the volume, issue and page numbers.

Link to publication

\footnotetext{
General rights rights.

- You may freely distribute the URL identifying the publication in the public portal. please follow below link for the End User Agreement:

www.umlib.nl/taverne-license

Take down policy

If you believe that this document breaches copyright please contact us at:

repository@maastrichtuniversity.nl

providing details and we will investigate your claim.
}

Copyright and moral rights for the publications made accessible in the public portal are retained by the authors and/or other copyright owners and it is a condition of accessing publications that users recognise and abide by the legal requirements associated with these

- Users may download and print one copy of any publication from the public portal for the purpose of private study or research.

- You may not further distribute the material or use it for any profit-making activity or commercial gain

If the publication is distributed under the terms of Article $25 f a$ of the Dutch Copyright Act, indicated by the "Taverne" license above, 
TH-302 in Combination with Radiotherapy Enhances the Therapeutic Outcome and Is Associated with Pretreatment $\left[{ }^{18} \mathrm{~F}\right] \mathrm{HX} 4 \mathrm{Hypoxia}$ PET Imaging $\mathbb{Q}$

\author{
Sarah G.J.A. Peeters ${ }^{1}$, Catharina M.L. Zegers ${ }^{1}$, Rianne Biemans' ${ }^{1}$ Natasja G. Lieuwes ${ }^{1}$, \\ Ruud G.P.M. van Stiphout ${ }^{1}$, Ala Yaromina ${ }^{1}$, Jessica D. Sun ${ }^{2}$, Charles P. Hart ${ }^{2}$, \\ Albert D. Windhorst ${ }^{3}$, Wouter van Elmpt', Ludwig J. Dubois ${ }^{1}$, and Philippe Lambin ${ }^{1}$
}

\title{
Abstract
}

Purpose: Conventional anticancer treatments are often impaired by the presence of hypoxia. TH-302 selectively targets hypoxic tumor regions, where it is converted into a cytotoxic agent. This study assessed the efficacy of the combination treatment of TH-302 and radiotherapy in two preclinical tumor models. The effect of oxygen modification on the combination treatment was evaluated and the effect of TH-302 on the hypoxic fraction (HF) was monitored using $\left[{ }^{18} \mathrm{~F}\right]$ HX4-PET imaging and pimonidazole IHC stainings.

Experimental Design: Rhabdomyosarcoma R1 and H460 NSCLC tumor-bearing animals were treated with TH-302 and radiotherapy ( $8 \mathrm{~Gy}$, single dose). The tumor oxygenation status was altered by exposing animals to carbogen ( $95 \%$ oxygen) and nicotinamide, $21 \%$ or $7 \%$ oxygen breathing during the course of the treatment. Tumor growth and treatment toxicity were monitored until the tumor reached four times its start volume $(\mathrm{T} 4 \times \mathrm{SV})$.
Results: Both tumor models showed a growth delay after TH-302 treatment, which further increased when combined with radiotherapy (enhancement ratio rhabdomyosarcoma 1.23; H460 1.49). TH-302 decreases the HF in both models, consistent with its hypoxia-targeting mechanism of action. Treatment efficacy was dependent on tumor oxygenation; increasing the tumor oxygen status abolished the effect of TH-302, whereas enhancing the HF enlarged TH-302's therapeutic effect. An association was observed in rhabdomyosarcoma tumors between the pretreatment HF as measured by $\left[{ }^{18} \mathrm{~F}\right] \mathrm{HX} 4-\mathrm{PET}$ imaging and the T4 $\times \mathrm{SV}$.

Conclusions: The combination of TH-302 and radiotherapy is promising and warrants clinical testing, preferably guided by the companion biomarker $\left[{ }^{18} \mathrm{~F}\right] \mathrm{HX} 4$ hypoxia PET imaging for patient selection. Clin Cancer Res; 21(13); 2984-92. (2015 AACR.

\section{Introduction}

Hypoxia is a common feature of solid tumors and is known to negatively influence treatment outcome $(1,2)$. Because of the disorganized vessel formation and consequently low oxygen concentrations, conventional chemotherapies and radiotherapies are less effective. To overcome hypoxia-induced treatment resistance, drugs have been developed that specifically target hypoxic

\footnotetext{
${ }^{1}$ Department of Radiation Oncology (MaastRO), GROW-School for Oncology and Developmental Biology, Maastricht University Medical Centre, Maastricht, the Netherlands. ${ }^{2}$ Threshold Pharmaceuticals, South San Francisco, California. ${ }^{3}$ Department of Radiology and Nuclear Medicine, VU University Medical Center, Amsterdam, the Netherlands.

Note: Supplementary data for this article are available at Clinical Cancer Research Online (http://clincancerres.aacrjournals.org/)

L.J. Dubois and P. Lambin contributed equally to this article.

Corresponding Author: Sarah G.J.A. Peeters, Department of Radiation Oncology (MaastRO Lab), GROW-School for Oncology and Developmental Biology, Maastricht University Medical Centre, UNS 50/23 PO Box 616, 6200 MD Maastricht, the Netherlands. Phone: 31-43-388-2908; Fax: 31-43-388-4540; E-mail: sarah.peeters@maastrichtuniversity.nl
}

doi: 10.1158/1078-0432.CCR-15-0018

(C)2015 American Association for Cancer Research. tumor regions. These so-called "hypoxia-activated prodrugs" (HAP) are nontoxic under normal oxygen concentrations but are activated in environments with low oxygen concentrations. TH-302 is a second-generation HAP of which the activation mechanism is based on the reduction of its 2-nitroimidazole moiety. Only in the presence of certain reductases under low oxygen concentrations is the toxic effector bromo-isophosphoramide mustard (Br-IPM) selectively released and able to crosslink DNA leading to cell death.

Preclinical studies have assessed the therapeutic effect of TH-302 alone or in combination with conventional chemotherapies in multiple xenograft models. TH-302 monotherapy led to reduced tumor growth in many of the xenograft models profiled, and was dependent on the hypoxic fraction (HF; ref. 3). Combining TH-302 with several clinically used chemotherapeutics offers advantage over single-agent treatment, although the treatment schedule and order of administration are of importance (4). A phase I study demonstrated the safety of TH-302 monotherapy in patients with solid malignancies (5). Other clinical phase I and II trials successfully combined the standard treatment doxorubicin with intravenous administration of TH-302 in patients with advanced soft tissue sarcoma $(6,7)$ and standard treatment gemcitabine with $\mathrm{TH}$ 302 in patients with advanced pancreatic cancer (8).

However, there are no published studies to assess the combination therapy of TH-302 and radiotherapy. Radiotherapy is one 


\section{Translational Relevance}

Radiotherapy is applied to $50 \%$ of all cancer patients and is therefore an important cancer treatment modality. Hypoxia is a feature of solid tumors that gives the opportunity of a tumortargeted approach. TH-302 is shown to be a promising hypoxia-activated prodrug. Several clinical trials have already demonstrated the applicability of TH-302 as a monotherapy and in combination with chemotherapy. In this study, the efficacy of the combination treatment of TH-302 and radiotherapy was assessed in two preclinical tumor models: the rat rhabdomyosarcoma model and the human non-small cell lung cancer H460 xenograft model. Furthermore, we monitored tumor hypoxia with an imaging biomarker used in clinical trials. Upon TH-302 treatment, the hypoxic cells, which are less sensitive to conventional anticancer therapies, were significantly decreased. We believe that the current study gives important directions for future clinical studies.

of the conventional treatment options applied to multiple cancer types and tumor hypoxia is a known radioresistance factor. The combination of TH-302 and radiotherapy is hypothesized to be complementary; where TH-302 specifically targets hypoxic cells, radiotherapy has the highest therapeutic gain in the well-oxygenated cells.

PET imaging is a noninvasive method characterizing the tumor oxygenation status in a three-dimensional manner. Several PET tracers have been developed that specifically visualize hypoxic regions. One of those tracers, based on the same 2-nitroimidazole hypoxia sensing mechanism as TH-302, is $\left[{ }^{18} \mathrm{~F}\right] \mathrm{HX} 4$. Preclinical and clinical trials have shown that $\left[{ }^{18} \mathrm{~F}\right] \mathrm{HX} 4$ is a reliable tool for the noninvasive detection of hypoxic tumor regions (9-11). Because TH-302, like tirapazamine, is expected to have only a therapeutic effect when hypoxic regions are present in the tumor (12), $\left[{ }^{18} \mathrm{~F}\right] \mathrm{HX} 4$ PET imaging may function as a useful predictive biomarker.

In the current study, we investigated the treatment effect of TH302 in combination with radiotherapy in two preclinical tumor models. We hypothesize that this combination therapy will enhance the therapeutic effect. Furthermore, we investigate the causal relationship between TH-302 efficacy and the modified tumor oxygenation status which was assessed before and after treatment using $\left[{ }^{18} \mathrm{~F}\right] \mathrm{HX} 4$ hypoxia PET imaging and only after treatment with pimonidazole staining. We hypothesize that the pretreatment hypoxia $\left[{ }^{18} \mathrm{~F}\right] \mathrm{HX} 4 \mathrm{PET}$ imaging will correlate with the treatment outcome.

\section{Materials and Methods}

Animal, tumor models, and treatment schedules

All animal experimental procedures were approved by the Animal Ethical Committee of Maastricht University (Maastricht, the Netherlands) and were in accordance with the Helsinki Declaration of 1975 as revised in 2000. All animals were monitored at least three times a week and their tumor volume was calculated using the formula: $\mathrm{A} \times \mathrm{B} \times \mathrm{C} \times \pi / 6$, in which $\mathrm{A}$, $\mathrm{B}$, and $\mathrm{C}$ are the three orthogonal diameters of the tumor as measured using a Vernier caliper, each corrected for the thickness of the skin.
Animals were randomized into the different treatment groups (Supplementary Fig. S1A) and were monitored until four times start volume $(\mathrm{T} 4 \times \mathrm{SV})$ was reached. For this calculation, the start volume of the first day of TH-302 treatment was used and fitting of the data was based on the regrowth phase. TH-302 was supplied by Threshold Pharmaceuticals and dissolved in $0.9 \% \mathrm{NaCl}$ to a concentration of $5 \mathrm{mg} / \mathrm{mL}$.

\section{Experimental models}

Syngeneic rhabdomyosarcoma R1 tumors $\left(1 \mathrm{~mm}^{3}\right)$ were implanted subcutaneously in the lateral flank of adult WAG/Rij rats. Experiments were started upon a mean tumor volume of $4.2 \mathrm{~cm}^{3}$ (range, 2.0-8.1) to ensure a stable HF. Treatment was administered on 4 consecutive days (Supplementary Fig. S1A) and consisted of an intraperitoneal injection (i.p.; $\mathrm{QD} \times 4$ ) with either $\mathrm{NaCl}$ or TH-302 $(25,50$, or $75 \mathrm{mg} / \mathrm{kg})$. Before the start of treatment, a PET scan was made using $\left[{ }^{18} \mathrm{~F}\right] \mathrm{HX} 4$. Radiotherapy (Varian Truebeam linear accelerator; $15 \mathrm{MeV}$ electrons) was applied in a single dose of $0,4,8$, or 12 Gy on day 3 of the treatment, 3 hours after $\mathrm{NaCl}$ or TH-302 injection, 1 hour after oxygen modification. During both PET imaging and radiotherapy, rats were anesthetized using a mixture of ketamine/xylazine (i.p; 66.7 and $6.7 \mathrm{mg} / \mathrm{kg}$, respectively). During the 5 days of treatment ( 1 day PET imaging, 4 days of injections with TH-302 or vehicle), animals were exposed to modified oxygen concentrations for 4 hours per day in order to alter the HF of the tumor. The combination oxygen modification of nicotinamide (i.p. $500 \mathrm{mg} / \mathrm{kg}$ ) and carbogen ( $95 \%$ oxygen, $5 \% \mathrm{CO}_{2} ; 5 \mathrm{~L} /$ minute) consisted of a nicotinamide injection and 30 minutes later the exposure to carbogen breathing for 3.5 hours. In the middle of the nicotinamide/carbogen treatment, $\mathrm{NaCl} / \mathrm{TH}-302$ was administered. Reduced oxygen breathing $\left(7 \%\right.$, residual $\mathrm{N}_{2} ; 2.5 \mathrm{~L} /$ minute) was given for 4 hours with the NaCl/TH-302 injection after the first 2 hours. The injection of the $\left[{ }^{18} \mathrm{~F}\right] \mathrm{HX} 4$ PET tracer [mean 18.8 MBq, range 7.1-25.1 MBq; lateral tail vein using an intravenous line (Venoflux $0.4 \mathrm{~mm} \mathrm{G27)}$ flushed with $10 \%$ heparine)] was given 2 hours before the end of the oxygen modification. PET imaging was performed 3 hours after tracer injection, as previously assessed (13). $\left[{ }^{18} \mathrm{~F}\right] \mathrm{HX} 4$ PET scans could not be performed on all treated animals due to production and supply difficulties. For the histologic control animals, PET imaging was also performed on day 4 of the treatment.

H460 lung carcinoma cells were resuspended $\left(1 \times 10^{6}\right.$ cells $/ \mathrm{mL}$; ATCC HTB-177) in Basement Membrane Matrix (Matrigel BD Biosciences) and injected in the lateral flank of NU-Foxn1-nu (NU/NU) mice. Experiments were started upon animals reaching a mean tumor volume of $225 \mathrm{~mm}^{3}$ (range $89-273 \mathrm{~mm}^{3}$ ). Mice were treated with either $\mathrm{NaCl}$ or $\mathrm{TH}-302(50 \mathrm{mg} / \mathrm{kg})$ for 5 consecutive days $(\mathrm{QD} \times 5)$. Treatment was combined with radiotherapy in a single dose of 0 or 8 Gy on day 4 for which the animals were anesthetized using a mixture of ketamine/xylazine (i.p; 66.7 and $6.7 \mathrm{mg} / \mathrm{kg}$, respectively). During the 5 days of treatment, animals were exposed to different oxygen concentrations; either a combination of nicotinamide $(500 \mathrm{mg} / \mathrm{kg}$ i.p.) and carbogen breathing ( $95 \%$ oxygen, $5 \%$ CO 2 ), $21 \%$ oxygen breathing (pressured air) or $7 \%$ oxygen breathing (residual $\mathrm{N}_{2}$ ). Total treatment time for all oxygen breathing schedules was 2.5 hours with the NaCl/TH-302 injection given 1 hour after the start of the treatment. Nicotinamide was administered 30 minutes before carbogen breathing was started, which was then applied for another 2 hours. Radiotherapy was given within 1 hour after the oxygen treatment. Histologic controls were administered with 
pimonidazole (60 mg/kg, i.p.; Hypoxyprobe kit, HP3-1000; Bio-connect) and Hoechst 33342 (15 mg/kg, i.v.; Sigma-Aldrich) 1 hour and 1 minute before sacrificing, respectively, followed by excision of the tumors that were snap frozen in liquid nitrogen and stored at -80 degrees Celsius until being processed.

\section{PET image acquisition and analysis}

Tracer synthesis of $\left[{ }^{18} \mathrm{~F}\right] \mathrm{HX} 4$ was performed as described previously (14). $\left[{ }^{18} \mathrm{~F}\right] \mathrm{HX} 4$ PET images were acquired and analyzed using a clinical PET/CT scanner (Siemens Biograph 40, Siemens Healthcare) as previously described (13). A volume of interest in the heart (sphere with a radius of $3 \mathrm{~mm}$ ) was defined as background region. The threshold to define the HF was set at 4.5 times the background uptake, because this method results in a HF that is in agreement with the pimonidazole staining-based results of a previous study on the rat rhabdomyosarcoma model (15).

\section{IHC staining and analysis}

Frozen H460 xenograft tumors were sectioned (5 $\mu \mathrm{m})$ and stained for hypoxia (pimonidazole), blood vessels (CD31) and perfusion (Hoechst 33342). Sections were fixed using cold acetone, rehydrated in TBS with $0.2 \%$ Tween-20 (TBST) and preincubated with normal goat serum before exposing them to the primary antibody rabbit anti-pimonidazole (1:150; HP3-1000, Bio-connect) and rat anti-mouse CD31 (1:500; BD biosciences). After washing with TBST, incubation with the secondary antibody goat anti-rabbit Alexa594 (1:500) and goat anti-rat Alexa488 (1:750, both Invitrogen) was performed. Sections were mounted using fluorescent mounting medium (DakoCytomation) and scanned for pimonidazole, blood vessels, and perfusion. After scanning, sections were stained for hematoxylin and eosin $(\mathrm{H} \& \mathrm{E})$.

Images were acquired using an Olympus BX51WI microscope equipped with a Hamamatsu EM-CCD C9100 digital camera, a motorized stage (Ludl Mac 2000) and a $10 \times$ objective. Micromanager 1.4 software was used for automated image acquisition (16). ImageJ version 1.49e (http://rsb.info.nih.gov/ij/) was used to stitch the images and perform quantitative analyses. All image recordings and analyses were performed by an investigator blinded to the subject coding. Viable tumor tissue was first delineated manually by excluding epidermis, stroma, and necrotic tumor regions based on $\mathrm{H} \& \mathrm{E}$ staining. The thresholds were set manually by two independent observers to discriminate signal from background. Finally the relative HF, microvessel density, and perfusion were calculated by determining the positive fraction within the viable tumor area.

\section{Statistical analysis}

GraphPad Prism software (version 5.01 for Windows) was used to perform statistical analyses. To determine the statistical significance of differences between two independent groups of variables, we used an unpaired $t$ test, whereas for matched groups, a paired $t$ test was applied. A two-way ANOVA was performed in $\mathrm{R}$ v3.0.1 to determine synergistic effects. $P$ values $<0.05$ were considered to be significant.

\section{Results}

Combination of TH-302 and radiotherapy

The effect of TH-302 in combination with radiotherapy was assessed in two tumor models, a rhabdomyosarcoma rat syngeneic model and a H460 human non-small cell lung cancer (NSCLC) xenograft mouse model. The treatment dose of TH-302 for rhabdomyosarcoma was assessed in a "tolerability" study, showing $25 \mathrm{mg} / \mathrm{kg}(\mathrm{QD} \times 4)$ to be the most optimal dose without adverse effects (Supplementary Fig. $\mathrm{S} 2$ ). This dose was therefore selected for further experiments. The TH-302 treatment dose of $50 \mathrm{mg} / \mathrm{kg}(\mathrm{QD} \times 5)$ for $\mathrm{H} 460$ was based on literature (3).

In both tumor models, TH-302 treatment showed an inhibition of the tumor growth, which was further reduced when TH-302 administration was combined with a single dose of radiotherapy ( 8 Gy; Fig. 1A). The time to reach four times start volume (T4 $\times$ SV, Fig. 1B) was significantly delayed upon TH-302 monotherapy from $12.4 \pm 1.7$ (mean $\pm \mathrm{SD}$ ) to $20.4 \pm 3.5$ days for rhabdomyosarcoma $(P<0.001)$ and $7.1 \pm 2.4$ to $13.6 \pm 4.8$ days for $\mathrm{H} 460(P=0.003)$. Compared with radiotherapy alone, $\mathrm{T} 4 \times \mathrm{SV}$ for the combination treatment was delayed from $24.9 \pm 3.0$ to $30.8 \pm 5.9$ for rhabdomyosarcoma $(P=0.026)$ and from $16.9 \pm$ 7.1 to $25.2 \pm 4.9$ for $\mathrm{H} 460(P=0.014)$, resulting in an enhancement ratio (ER) of 1.23 and 1.49, respectively (Fig. $1 \mathrm{~B}$ and Supplementary Tables S1 and S2). In addition, the effect of TH-302 was also assessed in the rhabdomyosarcoma model in combination with 4 and 12 Gy of radiotherapy, leading to an ER of 1.28 and 1.59, respectively (Supplementary Fig. S3A and S3B and Supplementary Table S1). TH-302 has a radiosensitizing effect in both tumor models and all radiotherapy doses. Moreover, the effect of the combination therapy TH-302 and 12 Gy radiotherapy in the rhabdomyosarcoma model was even synergistic.

\section{Hypoxic fraction}

The effect of TH-302 on the HF in the rhabdomyosarcoma model was assessed using $\left[{ }^{18} \mathrm{~F}\right] \mathrm{HX} 4$ hypoxia PET imaging and revealed a significant decrease from a baseline of $23.1 \% \pm 6.7 \%$ to $2.5 \% \pm 4.2 \%$ after TH-302 treatment $(P<0.001)$. NaCl treatment, as expected, did not affect the HF (Fig. 1C). In the H460 model, the HF was assessed immediately after the treatment using histologic controls injected with pimonidazole. The HF in subjects treated with TH-302 significantly decreased compared with the control animals ( $\mathrm{NaCl}: 7.8 \% \pm 3.0 \%$; TH-302: $1.3 \% \pm 0.5 \%$; Fig. 1D).

\section{Effect of oxygen modification on the efficacy of TH-302 and} radiotherapy

To investigate whether a causal relationship exists between $\mathrm{TH}$ 302 efficacy and tumor oxygenation, the amount of oxygen present in the tumor was modified on the days of TH-302 treatment by $7 \%$ or $95 \%$ oxygen breathing to increase or decrease the HF, respectively. Oxygen modification was performed 1 day in advance for the rhabdomyosarcoma model in order to assess the effect of this modification on the baseline HF using $\left[{ }^{18} \mathrm{~F}\right]$ HX4-PET imaging. Ambient air breathing animals had a HF of $22.2 \% \pm 13.8 \%$. A significant reduction in the HF to $2.1 \%$ $\pm 4.7 \%$ was seen after $95 \%$ oxygen breathing $(P<0.001)$, whereas $7 \%$ oxygen breathing significantly increased the HF to $29.5 \% \pm 14.7 \%(P=0.029$; Fig. $2 A)$. Exposing rhabdomyosarcoma-bearing rats to increasing oxygen conditions abolished the effect of TH-302 and reduced the T4 $\times$ SV from $20.4 \pm 3.5$ to $15.3 \pm 2.5$ days $(P=0.007$, Fig. $2 B$; Supplementary Fig. S3C and Supplementary Table S1), whereas control animals had an increased $\mathrm{T} 4 \times \mathrm{SV}$. Upon combination with radiotherapy, the 
Figure 1.

The combination of TH-302 and radiotherapy (8 Gy) reduces tumor growth in a rhabdomyosarcoma $(n=$ 8) and H460 $(n \geq 8)$ tumor model. A, growth curves; $B$, time to reach four times start volume $(\mathrm{T} 4 \times \mathrm{SV})$. Animals were treated with either control $(\mathrm{NaCl})$ or $\mathrm{TH}-302$ administered to the rhabdomyosarcoma model for 4 consecutive days with a dose of $25 \mathrm{mg} / \mathrm{kg}$ and for the $\mathrm{H} 460$ model for 5 consecutive days using a dose of $50 \mathrm{mg} / \mathrm{kg}$. Radiotherapy was performed on either the third or fourth day of TH-302 treatment. The HF of the control animals and the animals treated with $\mathrm{TH}-302$ was analyzed in C for rhabdomyosarcoma $(n=6)$ both before and immediately after treatment using $\left[{ }^{18} \mathrm{~F}\right] \mathrm{HX} 4$ hypoxia PET imaging (top, representative $\left[{ }^{18}\right.$ F]HX4 PET images with the delineation of the total tumor volume in black and the HF in red (bottom, quantification of $\mathrm{HF}$ per group) and in D for $\mathrm{H} 460(n=6)$ immediately after treatment using pimonidazole IHC staining [top, representative stainings, with hypoxia (pimonidazole) in green, perfusion (Hoechst) in blue, and vessels (CD31) in red]. The white scale bar indicates $500 \mu \mathrm{m}$. Bottom: quantification. Data, mean \pm SEM. ${ }^{*}, P<0.05 ;{ }^{* *}, P<0.005$ ${ }^{* * *}, P<0.001 ;{ }^{* * *}, P<0.0001$.
A

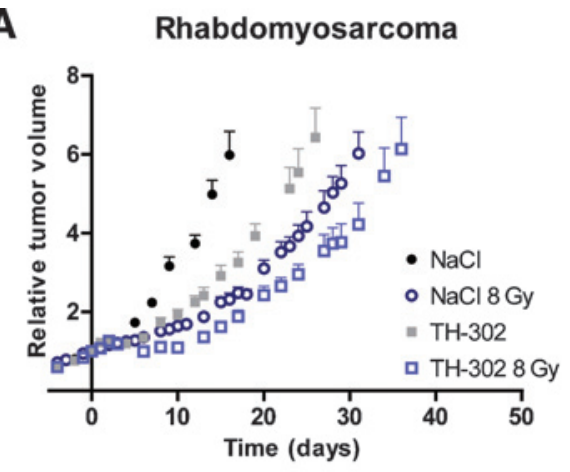

B

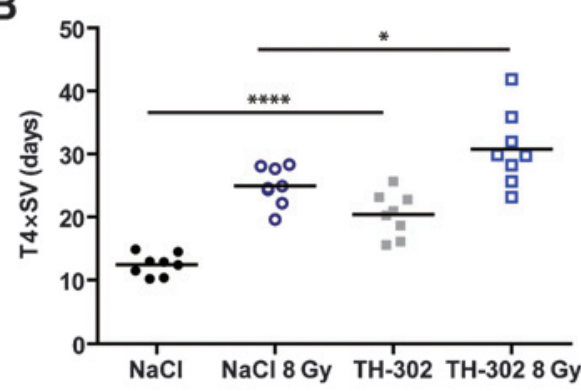

\section{C}
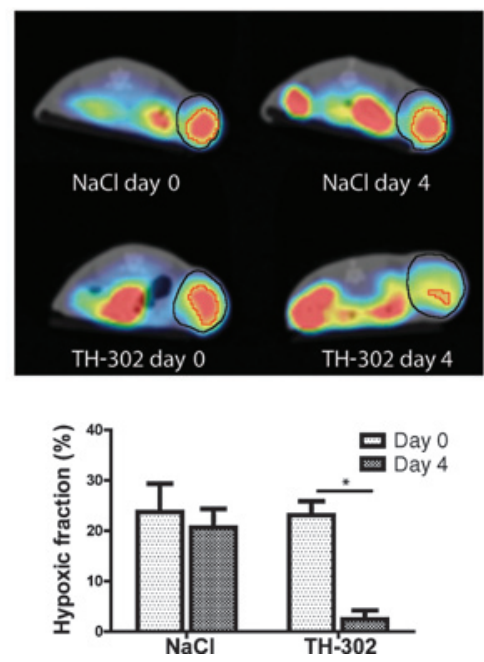

H460
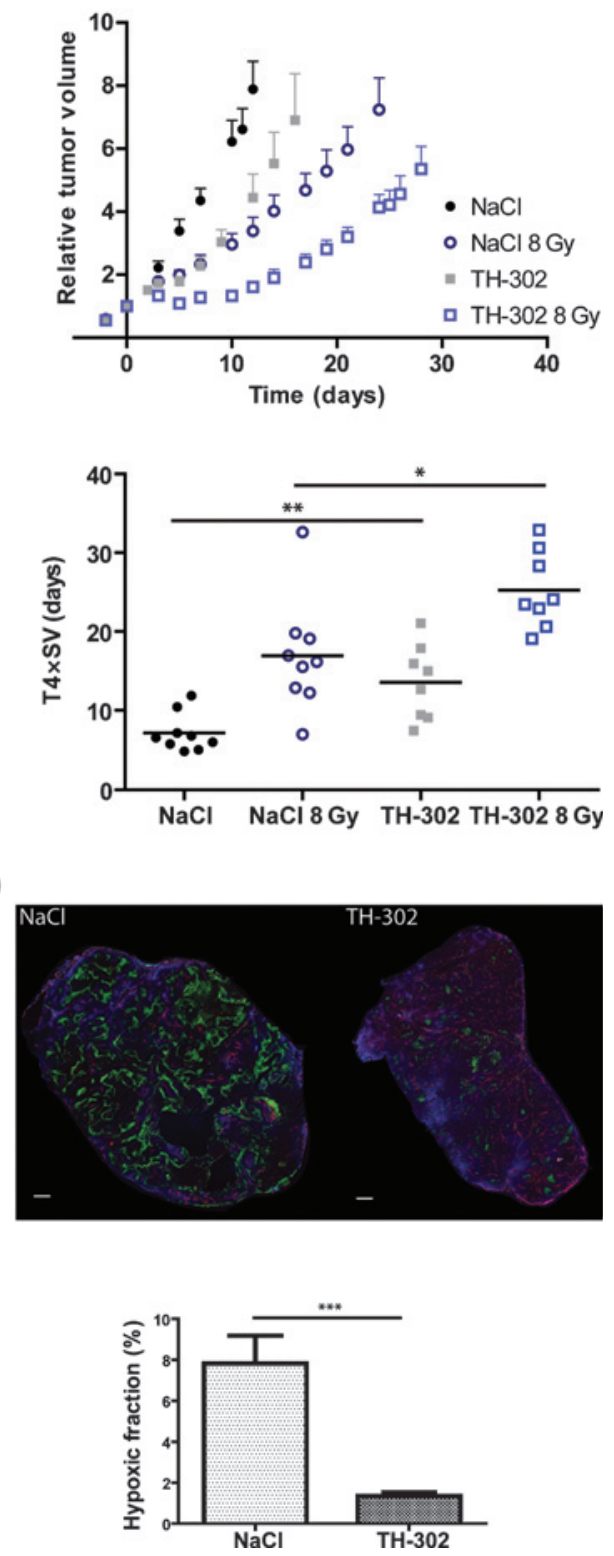

T4 $\times$ SV of TH-302-treated tumors decreased from $30.8 \pm 5.9$ (TH-302 + radiotherapy) to $25.7 \pm 2.9$ days (TH-302 + radiotherapy $+95 \% \mathrm{O}_{2}$ ). This is comparable with the $\mathrm{T} 4 \times \mathrm{SV}$ of $23.2 \pm 1.7$ days for animals treated with $\mathrm{NaCl}+$ radiotherapy under $95 \% \mathrm{O}_{2}$ conditions resulting in an ER of 1.11 (Supplementary Table S1). Exposing animals to $7 \%$ oxygen breathing resulted in a $\mathrm{T} 4 \times \mathrm{SV}$ of $22.6 \pm 4.2$ days for TH-302, which is significantly delayed compared with the animals treated with $\mathrm{NaCl}(\mathrm{T} 4 \times \mathrm{SV}: 16.1 \pm 1.9, P=0.001)$, although $7 \%$ oxygen treatment itself also had an effect on tumor growth in control animals. In the combination therapy of $7 \%$ oxygen breathing with radiotherapy, animals treated with TH-302 had a further reduction in the T $4 \times \mathrm{SV}$ to $35.4 \pm 6.1$ days with an ER of 1.29 compared with the animals treated with $\mathrm{NaCl}$ (Supplementary Fig. S3C and Supplementary Table S1).
Oxygen modification treatments were also applied to the H460 model where reducing the HF resulted in a decreased $\mathrm{T} 4 \times \mathrm{SV}$ from $25.2 \pm 4.9$ (TH-302 + radiotherapy) to $20.2 \pm 7.0$ (TH-302 + radiotherapy $+95 \% \mathrm{O}_{2}$ ) for the combination treatment. This decrease was not significant, however. The ER of TH-302 under high oxygen concentration remained stable at 1.50 versus 1.49 at $21 \% \mathrm{O}_{2}$ breathing. The tumor growth rate itself was unaffected by 95\% oxygen breathing (Fig. 2C and Supplementary Fig. S3D and Supplementary Table S2). Increasing the HF significantly enlarged the therapeutic potential of TH-302 compared with normal air breathing animals $(P=0.011)$, resulting in a $44 \times \mathrm{SV}$ of $22.7 \pm 7.9(\mathrm{~T} 4 \times \mathrm{SVTH}-302$ $21 \% \mathrm{O}_{2}: 13.6 \pm 4.8$ ). Although $7 \%$ oxygen breathing reduced the tumor growth slightly, radiotherapy increased the tumor growth of control animals under this condition. The effect of 

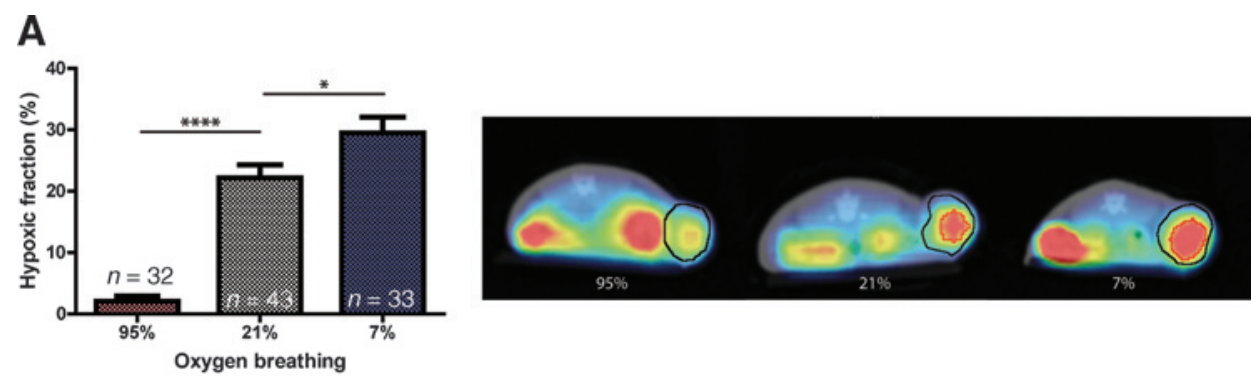

B

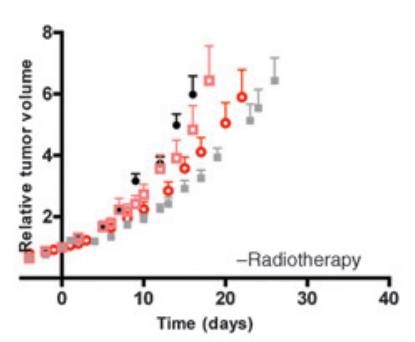

Rhabdomyosarcoma

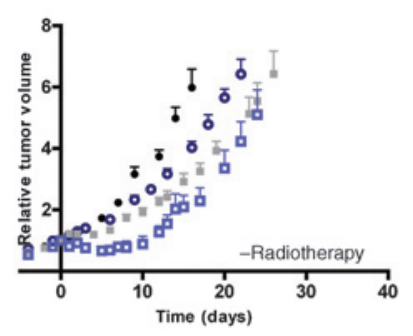

C
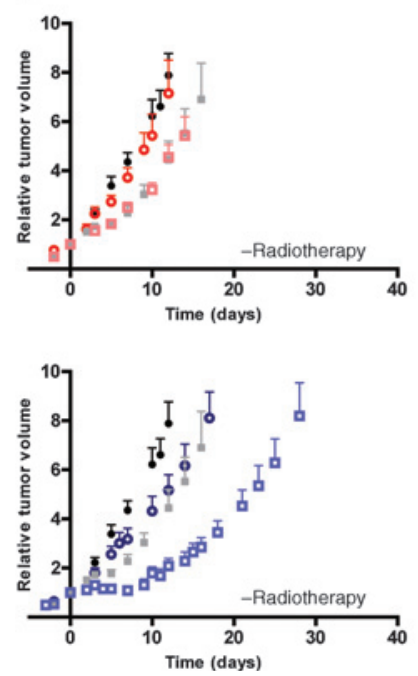

$$
\begin{aligned}
& \text { - } \mathrm{NaCl} \\
& \text { - } \mathrm{NaCl} 95 \% \\
& \text { = } \mathrm{TH}-302 \\
& \text { - } \mathrm{TH}-302 \text { 95\% }
\end{aligned}
$$

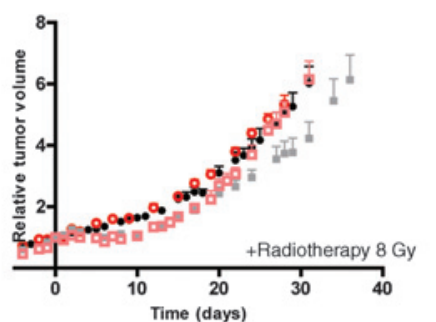

- $\mathrm{NaCl}$

- $\mathrm{NaCl} 7 \%$

= TH-302

- TH-302 $7 \%$

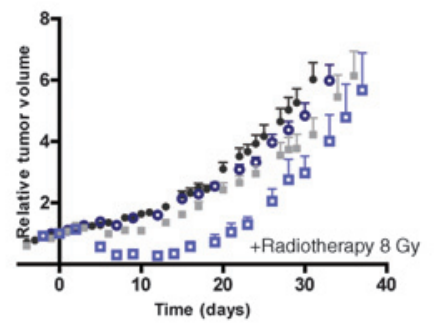

H460

- $\mathrm{NaCl}$

- $\mathrm{NaCl} 95 \%$

- $\mathrm{TH}-302$

ㅁ TH-302 95\%

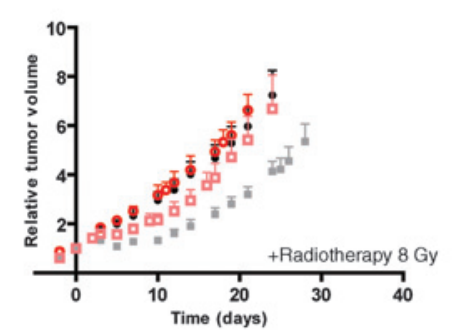

- $\mathrm{NaCl}$

- $\mathrm{NaCl} 7 \%$

- TH-302

a $\mathrm{TH}-3027 \%$

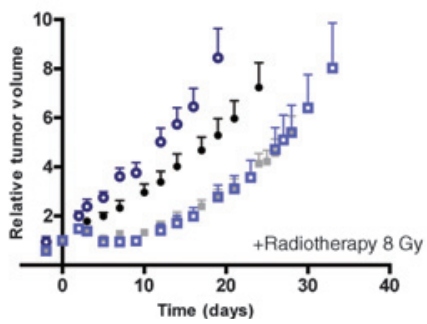

Figure 2.

The effect of oxygen modification on the combination treatment of $\mathrm{TH}-302$ and radiotherapy. $A$, exposing animals to $95 \%$ oxygen (nicotinamide $500 \mathrm{mg} / \mathrm{kg}$ i.p./carbogen breathing $95 \%$ oxygen, $5 \% \mathrm{CO}_{2}$ ), or $7 \%$ oxygen (residual $\mathrm{N}_{2}$ ) decreases or increases the HF, respectively, before the start of treatment compared with $21 \%$ oxygen breathing (ambient air). Representative $\left[{ }^{18} \mathrm{~F}\right] \mathrm{HX} 4 \mathrm{PET}$ images with the delineation of the tumor in black and the HF in red. ${ }^{*}, P<0.05$; ${ }_{* * * *}, P<0.0001$. Exposing the (B) rhabdomyosarcoma $(n \geq 7)$ or (C) H460 ( $n \geq 8$ ) model to modifying oxygen concentrations for the duration of the treatment for 4 and 2.5 hours per day, respectively. Data, mean \pm SEM. the TH-302 and radiotherapy combination increased to an ER of 2.45 for TH-302 + radiotherapy under low oxygen concentrations versus 1.49 for $\mathrm{TH}-302$ + radiotherapy under $21 \% \mathrm{O}_{2}$ concentrations (Supplementary Fig. S3D and Supplementary Table S2).

No toxic effects were observed for the different treatments in any of the groups as monitored by following changes in body weight (Supplementary Fig. S4A and S4B).
Oxygen modification and hypoxic fraction

To assess the effect of TH-302 treatment in combination with oxygen modification on the HF, a $\left[{ }^{18} \mathrm{~F}\right] \mathrm{HX} 4$ scan was acquired before and after treatment on the rhabdomyosarcoma histologic control animals. The HF of ambient air breathing animals decreased from $23 \% \pm 6.7 \%$ at baseline to $2.5 \% \pm 4.2 \%$ after TH-302 treatment. For $95 \%$ oxygen breathing animals, the HF was low before the start of the treatment, and this remained unchanged after 
either $\mathrm{NaCl}$ or TH-302 administration (Fig. 3A). The spread in HF of $7 \%$ oxygen breathing animals was very large. On average, the HF after treatment was lower than before treatment independent of $\mathrm{NaCl}$ or TH-302 treatment although this was not significant.

The HF in the H460 model was determined after the last TH302 injection using pimonidazole staining. TH-302 treatment significantly reduced the HF compared with the control animals (Figs. 1D and 3B). The different oxygen breathing conditions revealed a similar pattern; in combination with $95 \%$ oxygen breathing control, animals had a HF of $10.0 \% \pm 5.9 \%$, whereas animals treated with TH-302 had a HF of $2.1 \% \pm 1.0 \%$. Animals exposed to low oxygen concentrations in combination with $\mathrm{NaCl}$
Figure 3.

The effect of $\mathrm{TH}-302$ treatment and oxygen modification on the HF. A, HF was measured in the rhabdomyosarcoma model $(n=6)$ using $\left[{ }^{18} \mathrm{~F}\right] \mathrm{HX} 4$ hypoxia PET imaging the day before treatment and the last day of treatment with either control $(\mathrm{NaCl})$ or $\mathrm{TH}-302$ in combination with 95\% oxygen (nicotinamide and 95\% $\mathrm{O}_{2}$ breathing), ambient air, or $7 \%$ oxygen. B, pimonidazole staining was used to determine the HF after treatment in the $\mathrm{H} 460$ model. Top, a representative image is depicted per group. Bottom, quantification per group $(n=6) .{ }^{*}, P<0.05$; ${ }^{* *}, P<0.005$.
A

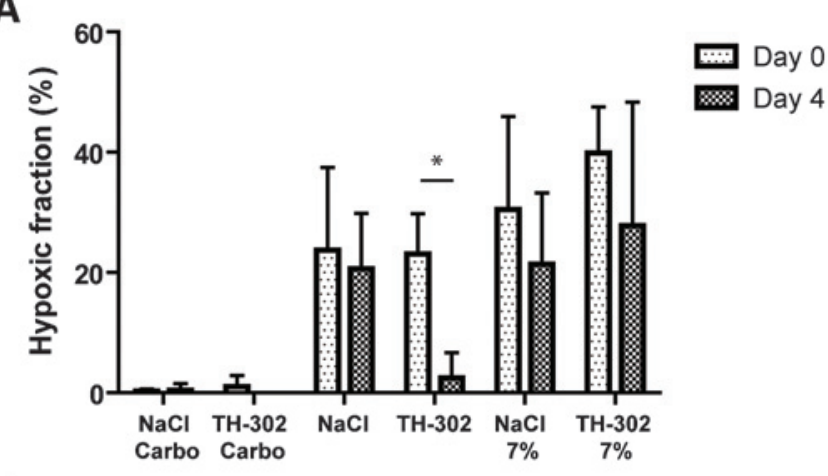

B
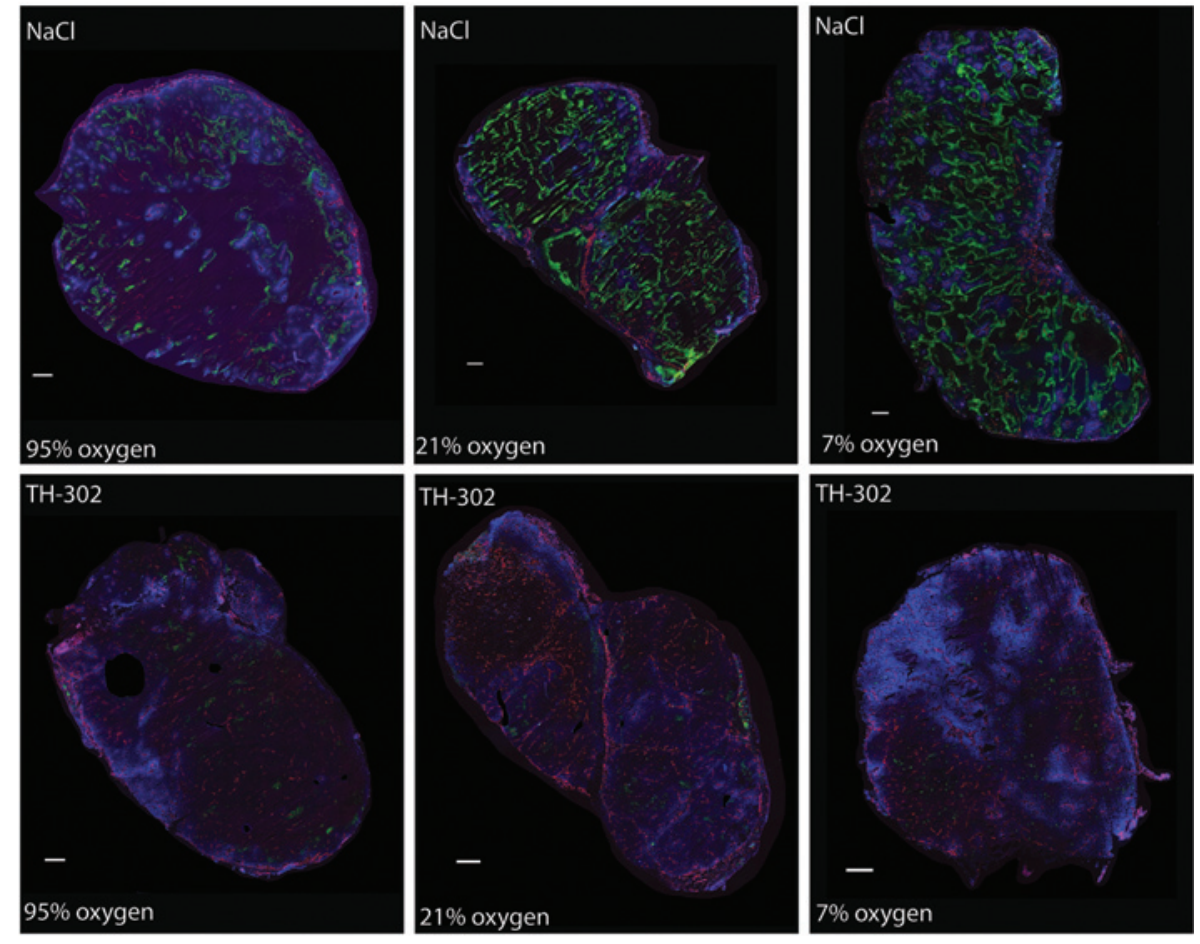

TH-302

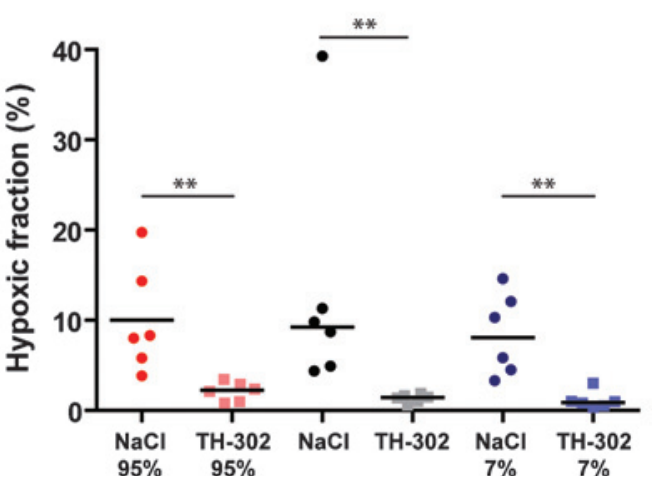


had a HF of $8.4 \% \pm 4.5 \%$, which was lower in the animals treated with TH-302 $(1.1 \% \pm 1.0 \%)$. Furthermore, TH-302-treated tumors had a decreased necrotic fraction, although this was only significant when animals were exposed to $21 \%$ oxygen. No differences were observed in the relative vessel area or perfusion (Supplementary Fig. S5).

Furthermore, we investigated whether the HF at the start of the therapy was associated with the treatment outcome expressed as $\mathrm{T} 4 \times \mathrm{SV}$. The T4 $\times$ SV for TH-302-treated tumors increased with increasing $\mathrm{HF}$ at onset meaning that $95 \%$ oxygen breathing animals reached their endpoint first, followed by ambient air and then $7 \%$ oxygen breathing animals (Fig. 4). The control animals, with or without radiotherapy, did not show this association.

\section{Discussion}

This study demonstrates the combination efficacy of the HAP TH-302 with radiotherapy in two preclinical tumor models, which was causally related to the tumor oxygen status. In addition, the $\left[{ }^{18} \mathrm{~F}\right] \mathrm{HX} 4$ determined HF was associated with the treatment outcome.

Pharmacokinetic studies in nontumor-bearing rats showed no adverse effects when the animals were treated with TH-302 (17). Although rhabdomyosarcoma-bearing rats showed no adverse effect after a $25 \mathrm{mg} / \mathrm{kg}$ TH-302 dose, dose-dependent adverse
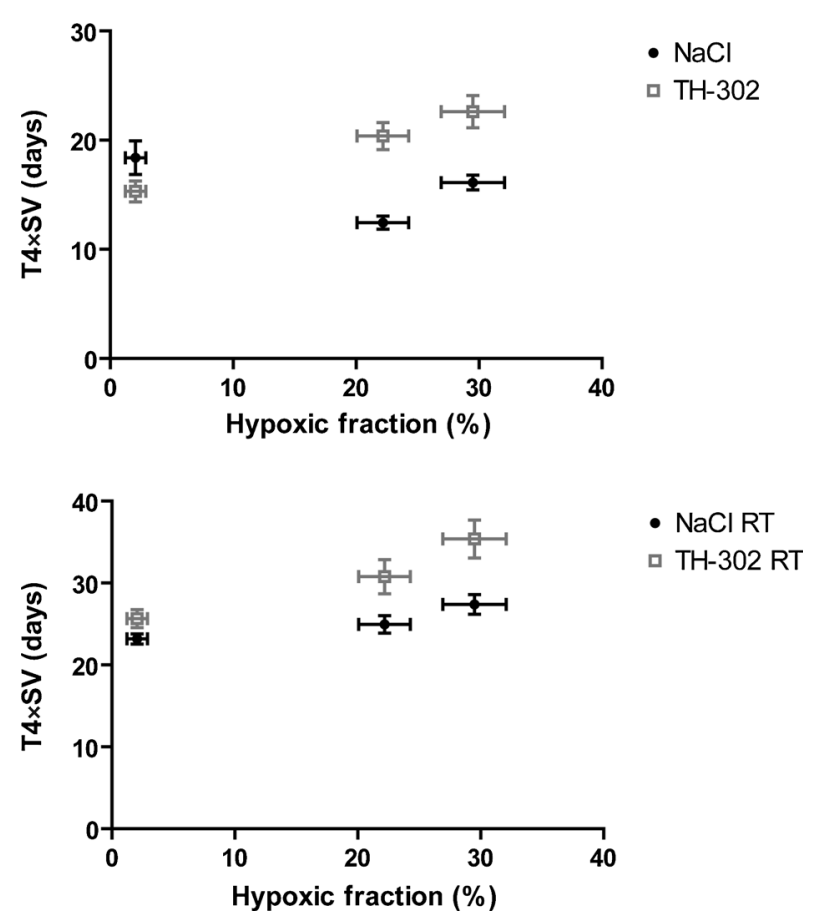

Figure 4.

The association between the pretreatment $\left[{ }^{18} \mathrm{~F}\right] \mathrm{HX} 4$-based $\mathrm{HF}$ and the treatment outcome in rhabdomyosarcoma tumors. HF (\%) as measured before the start of the treatment plotted to the time to reach four times the start volume $(\mathrm{T} 4 \times \mathrm{SV})$ for the control group $(\mathrm{NaCl})$ and animals treated with $\mathrm{TH}-302$ either with or without radiotherapy. HF is determined using $\left[{ }^{18} \mathrm{~F}\right] \mathrm{HX} 4$ hypoxia PET imaging after $95 \%$ oxygen breathing $(n=32)$, ambient air breathing $(n=43)$ and 7\% oxygen breathing $(n=33)$. The T4 $\times \mathrm{SV}$ is calculated for $\mathrm{NaCl}$ for all oxygen concentrations using 8 animals, TH-302 95\% oxygen $(n=7)$, ambient air and 7\% oxygen $(n=8)$. Data, mean \pm SEM. effects, such as a significant drop in body weight, diarrhea, and general malaise, were observed after higher dosing. Initial clinical studies also reported some adverse effects with skin and mucosal toxicities being dose limiting, while common adverse events were nausea, skin rash, fatigue, and vomiting $(5,6)$.

In this study, two different methods were used to assess the HF: noninvasive, clinically used $\left[{ }^{18} \mathrm{~F}\right] \mathrm{HX} 4$ PET imaging $(11,13)$, and IHC of pimonidazole adducts (9). Although $\left[{ }^{18} \mathrm{~F}\right] \mathrm{HX} 4$ hypoxia PET imaging represents the whole tumor in a noninvasive, reproducible, three-dimensional manner, IHC stainings can, in addition to hypoxia, extract more tumor microenvironmental information from the same tumor section. In both techniques, $\left[{ }^{18} \mathrm{~F}\right] \mathrm{HX} 4$ and pimonidazole are reduced under low oxygen concentrations (18) and a significant colocalization relationship was demonstrated at the tumor subregional level (9). Although monitored by different techniques, these data indicate that TH-302 has the same reducing effect on the HF in both tumor models. Furthermore, based on these data, $\left[{ }^{18} \mathrm{~F}\right] \mathrm{HX} 4$ imaging could be used as biomarker of response in a window-of-opportunity clinical trial. By performing a pretreatment scan, the initial tumor HF can be determined, followed by a single injection of TH-302. A posttreatment $\left[{ }^{18} \mathrm{~F}\right] \mathrm{HX} 4$ hypoxia scan can assess whether there is a response in HF to this HAP, without interference of other anticancer therapies. This new approach is designed to test new molecular entities in a clinical trial while being cost and patient efficient $(19,20)$.

A correlation has previously been reported between the HF and the tumor growth inhibition in xenograft models (3). This endorses our findings of a pronounced effect of TH-302 because the preclinical tumor models in this study were observed to have respectively somewhat higher and lower $\mathrm{HF}$ than the reported $\mathrm{HF}$ of $18.6 \%$ for rhabdomyosarcoma (15) and $16.3 \%$ for H460 (3). Although the HF in tumors was significantly decreased after TH302 treatment, not all hypoxic cells were eliminated, as observed in both tumor models. The remaining hypoxic cells might be resistant, unreachable by TH-302, or caused by cycling hypoxia. In addition to the decrease in the HF, this study shows a decrease in the necrotic fraction of the TH-302-treated tumors. This indicates that the dead cells are resorbed, which is supported by the stagnation in tumor growth after 3 days of TH-302 treatment. Other microenvironmental characteristics like relative vessel area and perfusion were not affected by TH-302 treatment, suggesting that the tumor maintains its vasculature. This is in agreement with previously published results on solid tumors (3).

Although TH-302 exhibits antitumor effects as a monotherapy, it has been shown that its therapeutic efficacy increases when combined with conventional anticancer therapies mainly targeting the nonhypoxic cells. On the basis of favorable outcomes of two clinical phase II trials $(7,8)$, phase III trials in metastatic or locally advanced unresectable pancreatic adenocarcinoma (NCT01746979) and advanced soft-tissue sarcoma (NCT01440088) are currently ongoing. However, to our knowledge, no study has investigated the combination treatment of TH-302 with radiotherapy and $\left[{ }^{18} \mathrm{~F}\right] \mathrm{HX} 4$ hypoxia imaging. This combination is thought to be effective especially because radiotherapy can be locally applied, specifically targeting the tumor while preserving normal tissue. In this study, we show that this combination is effective and causes a delayed tumor growth and increased $\mathrm{T} 4 \times \mathrm{SV}$ for both investigated tumor models, confirming the hypothesis that the combination therapy of TH-302 and radiotherapy will lead to an enhanced therapeutic effect. In the 
rhabdomyosarcoma tumor model, TH-302 treatment was combined with a single dose of 4,8 , and 12 Gy of radiotherapy resulting in a dose-dependent effect. In subsequent studies, the single radiotherapy dose of 8 Gy was used, reasoning that the regrowth of the tumor solely depends on hypoxic cells (21), providing a basis for TH-302 efficacy. This approach is different from clinical practice where fractionated radiotherapy schedules are used. By applying $2 \mathrm{~Gy}$ fractions to the tumor, reoxygenation occurs and the HF gradually decreases $(22,23)$. We speculate that the combination of TH-302 with fractionated radiotherapy would also increase the therapeutic effect of the radiotherapy because the $\mathrm{HF}$ is reduced by the pretreatment of $\mathrm{TH}-302$, increasing the potential of radiotherapy.

In this study, we further wanted to elucidate whether TH-302 efficacy is dependent on the tumor oxygen status. Exposing animals to either nicotinamide and carbogen or $7 \%$ oxygen breathing has been demonstrated to be effective in modulating the HF in tumors $(9,24,25)$. Altering oxygen breathing before the TH-302 treatment did modify the tumor HF in rhabdomyosarcoma animals as measured by $\left[{ }^{18} \mathrm{~F}\right] \mathrm{HX} 4$. However, in the H460 model, the HF was determined only after TH-302 treatment and at this point no differences were observed in control animals of the various oxygen modifications. A possible explanation would be that the mice adapted to the oxygen breathing schedule, preventing the tumor HF from changing, which has been observed for mice exposed to long-term carbogen breathing $(26,27)$. In the rhabdomyosarcoma model, the tumor growth of control animals was significantly reduced upon oxygen modification as well as in the mice exposed to $7 \%$ oxygen breathing mice. No effect on tumor growth was observed after oxygen modification in another study using $\mathrm{H} 460$ tumors exposed to $95 \%$ or $10 \%$ oxygen breathing (3). This unexpected finding could possibly be explained by the stress induced by the exposure to the oxygen modifications, although TH-302 or radiotherapy treatment groups did not seem to be influenced by this. However, by calculating the enhancement ratio, these oxygen modification effects are taken into account. Despite these effects on growth delay, a positive effect of the therapy is observed. Radiotherapy was applied 1 hour after carbogen breathing without any beneficial effect. This can be explained by the fact that tumor oxygen concentrations return to pre-carbogen levels within 1 minute after stopping carbogen treatment as detected by Eppendorf electrode measurements (28). Furthermore, clinical studies have shown that the presence of hypoxia and the pretreatment selection of patients with hypoxic tumors is essential for the combination of nicotinamide administration and carbogen breathing to be effective $(29,30)$. Breathing low oxygen concentrations reduced the effect of radiotherapy in the H460 model, indicating that, although not detected on pimonidazole IHC staining, low oxygen concentrations counteracted the irradiation. The effect of TH-302 is abolished by carbogen breathing in the rhabdomyosarcoma model independent of radiotherapy. This can be explained by the reduced HF leaving almost no cells present to convert TH-302 into its cytotoxic metabolite. For the H460 model the HF did not decrease upon carbogen breathing what reflects in the unchanged tumor growth compared with control tumors. Upon radiotherapy, however, there is a trend toward a faster tumor growth that also indicates abolishment of the TH-302 efficacy. In tumors with an enlarged HF, TH-302 caused a slight, nonsignificant, delay in tumor growth compared to TH-302 under normal air conditions. Moreover, TH302 decreased the HF to almost zero under ambient air condi- tions, while with $7 \%$ oxygen breathing the HF is still $28 \%$. Although this result could be caused by the counteracting effects of TH-302 reducing the HF and the 7\% oxygen breathing increasing the $\mathrm{HF}$, we speculate that it is caused by a limited availability of TH-302 to target all hypoxic cells. In H460 tumors, 7\% oxygen breathing resulted in an increased therapeutic effect with an enhancement ratio of 2.2 for TH-302 alone and 2.5 for the combination therapy of TH-302 and radiotherapy. This result demonstrates that when sufficient TH-302 is present, more TH302 is reduced upon low oxygen concentrations, causing an increased cytotoxicity.

A causal relation between the pretreatment HF measured by $\left[{ }^{18} \mathrm{~F}\right] \mathrm{HX} 4$ and the TH-302 treatment outcome was observed. These results indicate that pretreatment evaluation of hypoxia could be a useful tool in selecting tumors that benefit from the additional hypoxia targeting treatment. This hypoxia-based patient selection could also be used in other therapy strategies for instance to target hypoxic subvolumes by escalate radiation dose (31). Furthermore, this information could be implemented in decision-support systems to predict tumor response and optimize patient therapy (32). These applications demonstrate the importance of gaining pretreatment information by hypoxia imaging.

\section{Conclusion}

This study demonstrates that TH-302 treatment together with conventional radiotherapy is a promising combination with an increased therapeutic potential, and warrants further testing. Furthermore, detecting the tumor HF by $\left[{ }^{18} \mathrm{~F}\right] \mathrm{HX} 4$ PET imaging may allow the ability to predict which patients will benefit most from the hypoxia targeted TH-302 treatment and gives the possibility to noninvasively monitor TH-302 efficacy in the context of window-of-opportunity trials. On the basis of this preclinical study, we suggest a clinical trial for treating patients with the combination of TH-302 and radiotherapy while monitoring the HF before and during the treatment.

\section{Disclosure of Potential Conflicts of Interest}

C.P. Hart has ownership interest (including patents) in Threshold Pharmaceuticals stock. No potential conflicts of interest were disclosed by the other authors.

\section{Authors' Contributions}

Conception and design: S.G.J.A. Peeters, J.D. Sun, A.D. Windhorst, L.J. Dubois, P. Lambin

Development of methodology: S.G.J.A. Peeters, R.G.P.M. van Stiphout, J.D. Sun, L.J. Dubois, P. Lambin

Acquisition of data (provided animals, acquired and managed patients, provided facilities, etc.): S.G.J.A. Peeters, R. Biemans, N.G. Lieuwes, A. Yaromina, A.D. Windhorst, L.J. Dubois

Analysis and interpretation of data (e.g., statistical analysis, biostatistics, computational analysis): S.G.J.A. Peeters, C.M.L. Zegers, N.G. Lieuwes, R.G.P. M. van Stiphout, A. Yaromina, W. van Elmpt, L.J. Dubois, P. Lambin

Writing, review, and/or revision of the manuscript: S.G.J.A. Peeters, C.M.L. Zegers, R.G.P.M. van Stiphout, A. Yaromina, C.P. Hart, A.D. Windhorst, W. van Elmpt, L.J. Dubois, P. Lambin

Administrative, technical, or material support (i.e., reporting or organizing data, constructing databases): S.G.J.A. Peeters, R. Biemans, N.G. Lieuwes, P. Lambin

Study supervision: L.J. Dubois, P. Lambin

\section{Grant Support}

This work was financially supported by the QuIC-ConCePT project, which is partly funded by EFPI A companies and the Innovative Medicine Initiative Joint 
Undertaking (IMI JU) under Grant Agreement No. 115151, and the EU 6th and 7 th framework program (METOXIA, EURECA, ARTFORCE), Kankeronderzoekfonds Limburg from the Health Foundation Limburg, and the Dutch Cancer Society (KWF UM 2011-5020, KWF-MAC 2011-4970, KWF MAC 2013-6425, KWF MAC 2013-6089). The micrographs in this paper were taken with a confocal spinning disk microscope financed by The Netherlands Organization for Scientific Research (NWO), grant number 911-06-003.

\section{References}

1. Horsman MR, Mortensen LS, Petersen JB, Busk M, Overgaard J. Imaging hypoxia to improve radiotherapy outcome. Nature reviews Clinical oncology 2012;9:674-87.

2. Vaupel P, Mayer A. Hypoxia in cancer: significance and impact on clinical outcome. Cancer Metastasis Rev 2007;26:225-39.

3. Sun JD, Liu Q, Wang J, Ahluwalia D, Ferraro D, Wang Y, et al. Selective tumor hypoxia targeting by hypoxia-activated prodrug TH-302 inhibits tumor growth in preclinical models of cancer. Clin Cancer Res 2012; 18:758-70.

4. Liu Q, Sun JD, Wang J, Ahluwalia D, Baker AF, Cranmer LD, et al. TH-302, a hypoxia-activated prodrug with broad in vivo preclinical combination therapy efficacy: optimization of dosing regimens and schedules. Cancer Chemother Pharmacol 2012;69:1487-98.

5. Weiss GJ, Infante JR, Chiorean EG, Borad MJ, Bendell JC, Molina JR, et al. Phase 1 study of the safety, tolerability, and pharmacokinetics of TH-302, a hypoxia-activated prodrug, in patients with advanced solid malignancies. Clin Cancer Res 2011;17:2997-3004.

6. Ganjoo KN, Cranmer LD, Butrynski JE, Rushing D, Adkins D, Okuno SH, et al. A phase I study of the safety and pharmacokinetics of the hypoxiaactivated prodrug TH-302 in combination with doxorubicin in patients with advanced soft tissue sarcoma. Oncology 2011;80:50-6.

7. Chawla SP, Cranmer LD, Van Tine BA, Reed DR, Okuno SH, Butrynski JE, et al. Phase II study of the safety and antitumor activity of the hypoxiaactivated prodrug TH-302 in combination with doxorubicin in patients with advanced soft tissue sarcoma. J Clin Oncol 2014;32:3299-306.

8. Borad MJ, Reddy SG, Bahary N, Uronis HE, Sigal D, Cohn AL, et al Randomized Phase II Trial of Gemcitabine Plus TH-302 Versus Gemcitabine in Patients With Advanced Pancreatic Cancer. J Clin Oncol 2014 Dec 15. [Epub ahead of print].

9. Dubois LJ, Lieuwes NG, Janssen MH, Peeters WJ, Windhorst AD, Walsh JC, et al. Preclinical evaluation and validation of $[18 \mathrm{~F}] \mathrm{HX} 4$, a promising hypoxia marker for PET imaging. Proc Natl Acad Sci U S A 2011;108: $14620-5$.

10. Zegers CM, van Elmpt W, Wierts R, Reymen B, Sharifi H, Ollers MC, et al. Hypoxia imaging with [(1)(8)F]HX4 PET in NSCLC patients: defining optimal imaging parameters. Radiother Oncol 2013;109:58-64.

11. Zegers CM, van Elmpt W, Reymen B, Even AJ, Troost EG, Ollers MC, et al. In vivo quantification of hypoxic and metabolic status of NSCLC tumors using $\left[{ }^{18} \mathrm{~F}\right] \mathrm{HX} 4$ and $\left[{ }^{18} \mathrm{~F}\right]$ FDG-PET/CT imaging. Clin Cancer Res 2014;20: 6389-97.

12. Rischin D, Hicks RJ, Fisher R, Binns D, Corry J, Porceddu S, et al. Prognostic significance of $[18 \mathrm{~F}]$-misonidazole positron emission tomographydetected tumor hypoxia in patients with advanced head and neck cancer randomly assigned to chemoradiation with or without tirapazamine: a substudy of Trans-Tasman Radiation Oncology Group Study 98.02. J Clin Oncol 2006;24:2098-104.

13. Peeters SG, Zegers CM, Lieuwes NG, van Elmpt W, Eriksson J, van Dongen GA, et al. A comparative study of the hypoxia PET tracers $\left[{ }^{18} \mathrm{~F}\right] \mathrm{HX} 4,\left[{ }^{18} \mathrm{~F}\right]$ FAZA, and $\left[{ }^{18} \mathrm{~F}\right] \mathrm{FMISO}$ in a preclinical tumor model. Int $\mathrm{J}$ Radiat Oncol Biol Phys 2015;91:351-9.

14. van Loon J, Janssen MH, Ollers M, Aerts HJ, Dubois L, Hochstenbag M, et al. PET imaging of hypoxia using [18F]HX4: a phase I trial. Eur J Nucl Med Mol Imaging 2010;37:1663-8.

15. Dubois L, Landuyt W, Haustermans K, Dupont P, Bormans G, Vermaelen P, et al. Evaluation of hypoxia in an experimental rat tumour model by [(18)
The costs of publication of this article were defrayed in part by the payment of page charges. This article must therefore be hereby marked advertisement in accordance with 18 U.S.C. Section 1734 solely to indicate this fact.

Received January 5, 2015; revised March 12, 2015; accepted March 12, 2015; published OnlineFirst March 24, 2015.

F]fluoromisonidazole PET and immunohistochemistry. Br J Cancer 2004;91:1947-54.

16. Edelstein A, Amodaj N, Hoover K, Vale R, Stuurman N. Computer control of microscopes using microManager. Curr Protoc Mol Biol 2010;Chapter 14:Unit14 20.

17. Jung D, Lin L, Jiao H, Cai X, Duan JX, Matteucci M. Pharmacokinetics of TH302: a hypoxically activated prodrug of bromo-isophosphoramide mustard in mice, rats, dogs and monkeys. Cancer Chemother Pharmacol 2012;69:643-54

18. Wilson WR, Hay MP. Targeting hypoxia in cancer therapy. Nat Rev Cancer 2011;11:393-410.

19. Fass L. Imaging and cancer: a review. Mol Oncol 2008;2:115-52.

20. Orloff J, Douglas F, Pinheiro J, Levinson S, Branson M, Chaturvedi P, et al. The future of drug development: advancing clinical trial design. Nat Rev Drug Discov 2009;8:949-57.

21. Hill RP, Bush RS, Yeung P. The effect of anaemia on the fraction of hypoxic cells in an experimental tumour. Br J Radiol 1971;44:299-304.

22. Stanley JA, Shipley WU, Steel GG. Influence of tumour size on hypoxic fraction and therapeutic sensitivity of Lewis lung tumour. Br J Cancer 1977;36:105-13.

23. Wouters BG, Brown JM. Cells at intermediate oxygen levels can be more important than the "hypoxic fraction" in determining tumor response to fractionated radiotherapy. Radiat Res 1997;147:541-50.

24. Horsman MR, Overgaard J. Preclinical studies on how to deal with patient intolerance to nicotinamide and carbogen. Radiother Oncol 2004;70:301-9.

25. Troost EG, Laverman P, Kaanders JH, Philippens M, Lok J, Oyen WJ, et al. Imaging hypoxia after oxygenation-modification: comparing [18F]FMISO autoradiography with pimonidazole immunohistochemistry in human xenograft tumors. Radiother Oncol 2006;80:157-64.

26. Hou H, Dong R, Lariviere JP, Mupparaju SP, Swartz HM, Khan N Synergistic combination of hyperoxygenation and radiotherapy by repeated assessments of tumor $\mathrm{pO} 2$ with EPR oximetry. J Radiat Res 2011;52:568-74

27. Khan N, Li H, Hou H, Lariviere JP, Gladstone DJ, Demidenko E, et al. Tissue pO2 of orthotopic 9L and C6 gliomas and tumor-specific response to radiotherapy and hyperoxygenation. Int J Radiat Oncol Biol Phys 2009; 73:878-85.

28. Martin L, Lartigau E, Weeger P, Lambin P, LeRidant AM, Lusinchi A, et al. Changes in the oxygenation of head and neck tumors during carbogen breathing. Radiother Oncol 1993;27:123-30.

29. Janssens GO, Rademakers SE, Terhaard CH, Doornaert PA, Bijl HP, van den Ende $\mathrm{P}$, et al. Accelerated radiotherapy with carbogen and nicotinamide for laryngeal cancer: results of a phase III randomized trial. J Clin Oncol 2012;30:1777-83.

30. Schuuring J, Bussink J, Bernsen HJ, Peeters W, van der Kogel AJ. Effect of carbogen breathing on the radiation response of a human glioblastoma xenograft: analysis of hypoxia and vascular parameters of regrowing tumors. Strahlenther Onkol 2006;182:408-14.

31. Bentzen SM, Gregoire V. Molecular imaging-based dose painting: a novel paradigm for radiation therapy prescription. Semin Radiat Oncol 2011; 21:101-10.

32. Lambin P, van Stiphout RG, Starmans MH, Rios-Velazquez E, Nalbantov G, Aerts HJ, et al. Predicting outcomes in radiation oncology-multifactorial decision support systems. Nat Rev Clin Oncol 2013;10:27-40. 


\section{Clinical Cancer Research}

\section{TH-302 in Combination with Radiotherapy Enhances the Therapeutic Outcome and Is Associated with Pretreatment [ 18 F]HX4 Hypoxia PET Imaging}

Sarah G.J.A. Peeters, Catharina M.L. Zegers, Rianne Biemans, et al.

Clin Cancer Res 2015;21:2984-2992. Published OnlineFirst March 24, 2015.

\section{Updated version Access the most recent version of this article at: doi:10.1158/1078-0432.CCR-15-0018}

Supplementary Access the most recent supplemental material at:

Material http://clincancerres.aacrjournals.org/content/suppl/2015/03/25/1078-0432.CCR-15-0018.DC1

Cited articles This article cites 30 articles, 8 of which you can access for free at: http://clincancerres.aacrjournals.org/content/21/13/2984.full\#ref-list-1

Citing articles This article has been cited by 13 HighWire-hosted articles. Access the articles at: http://clincancerres.aacrjournals.org/content/21/13/2984.full\#related-urls

E-mail alerts Sign up to receive free email-alerts related to this article or journal.

Reprints and Subscriptions

Permissions
To order reprints of this article or to subscribe to the journal, contact the AACR Publications Department at pubs@aacr.org.

To request permission to re-use all or part of this article, use this link http://clincancerres.aacrjournals.org/content/21/13/2984.

Click on "Request Permissions" which will take you to the Copyright Clearance Center's (CCC) Rightslink site. 